\title{
Under utilisation of the 2-week wait initiative for lung cancer by primary care and its effect on the urgent referral pathway
}

\author{
NR Lewis', I Le Jeune' and DR Baldwin*,I \\ 'Department of Respiratory Medicine, David Evans Centre, Nottingham City Hospital, Hucknall Road, Nottingham NG5 IPB, UK
}

\begin{abstract}
The '2-week wait' scheme for referral of patients with cancer to secondary care coincided with the introduction of Department of Health (DoH) Guidelines on referral of patients with suspected lung cancer. The aim of this study was to examine the impact of this process on the urgent referral pathway for lung cancer. Medical records of all patients referred with suspected lung cancer were reviewed for the year prior to introduction of the 2-week wait and DoH guidelines and for the subsequent 24 months. A total of 1044 patients were referred, of which 650 (62\%) were found to have malignancy. In the first and second years of the 2-week wait scheme, only 57 and 58\% were referred via the scheme. Department of Health guidelines were followed in all but a small number. Median wait time increased from 7 to 9 days. The proportion of all urgent referrals seen within 2 weeks fell from 84 to $71 \%$. The proportion of non-2-week wait urgent referrals being seen within 2 weeks was only $75.5 \%$ in the first year of the scheme and fell further to $60.9 \%$ in the second year. The absolute number of referrals rose and the proportion having cancer fell from $78 \%$ before the scheme to $46 \%$ in the second year. During this time, there was no change in stage at presentation. Symptoms were not helpful in discriminating benign from malignant disease and haemoptysis was actually more common in the benign group. However, over $50 \%$ of patients in the benign group were appropriate to be seen in secondary care. The 2-week wait scheme has so far failed to reduced waiting times for lung cancer. The findings of this study suggest that this is partly due to continued usage of urgent referral routes outside the 2-week wait scheme and secondly due to a large increase in referrals, probably generated by the introduction of the DoH guidelines. Some adjustment to the guidelines may be appropriate to reflect more emphasis on the early performance of a chest X-ray and the use of direct access to other imaging modalities such as CT. Patients referred outside the 2-week wait are disadvantaged and thus practitioners would be wise to refer all their patients through the 2-week wait system.

British Journal of Cancer (2005) 93, 905-908. doi: I 0.1038/sj.bjc.6602798 www.bjcancer.com
\end{abstract}

Published online 27 September 2005

(c) 2005 Cancer Research UK

Keywords: lung cancer; waiting times; diagnosis; investigation; symptoms

The Government white paper The New NHS - Modern, Dependable (Department of Health National Standards, Local Action, 2004) proposed that everyone with suspected cancer should be given the opportunity to be seen by a specialist within 2 weeks of the date of the GP referral. In order to introduce this '2-week wait' principle to lung cancer, the Department of Health (DoH) introduced national guidelines for the urgent referral of suspected lung carcinoma in April 2000. The intention was to simplify and streamline the referral process from primary care to respiratory physicians, thus expediting treatment by specialist multidisciplinary units (Department of Health NHS Improvement Plan June, 2004).

Here, we report on the utilisation and impact of this process on the urgent referral pathway for lung cancer.

\section{MATERIALS AND METHODS}

Medical records of all patients referred with suspected lung cancer to Nottingham City Hospital in the 12 months prior to the

\footnotetext{
*Correspondence: Dr DR Baldwin;

E-mail: david.baldwin@nottingham.ac.uk

Revised I August 2005; accepted 22 August 2005; published online 27 September 2005
}

introduction of DoH guidelines (1st April 1999-31st March 2000) and the subsequent 24 months (1st April 2000-31st March 2002) were retrospectively audited by three observers (NRL, DRB, ILJ). The reason for referral, referral route and times from GP referral to first hospital visit, investigations, diagnosis and treatment were recorded along with outcome data including surgical stage histological diagnosis, management plan and treatment modality. Statistical comparisons for these nonparametric data were made using the Mann-Whitney $U$-test. Fisher's exact test and $\chi^{2}$ tests were used for comparisons of proportions.

\section{RESULTS}

\section{Number of patients referred and referral route}

A total of 1044 patients were referred to the respiratory physicians with a suspected diagnosis of lung cancer during the 3-year audit period. In all, $628(60.2 \%)$ were male and the median age was 73 years (range 24-98 years). Malignancy was confirmed in 650 (62.3\%), of which $578(88.9 \%)$ were found to have a primary lung tumour. Of these $465(80.4 \%)$ were non-small-cell carcinoma, 86 (14.9\%) small-cell carcinoma, $22(3.8 \%)$ mesothelioma and five $(0.9 \%)$ were other tumours. 
Table 1 shows the number of referrals for each year. Only $57 \%$ of patients were referred via the 2 -week wait scheme (201/352) in $2000 / 2001$ and $58 \%(236 / 404)$ in $2001 / 2002$.

\section{Adherence to DoH guidelines for referral}

Only four patients ( $1 \%$ of referrals) in the first year after introduction of the guidelines, and 16 patients (4\% of referrals) in the second year failed to meet the criteria for urgent referral listed in the DoH guidelines.

\section{Effect of introduction of 2-week wait and DoH guidelines on waiting times}

Table 2 shows the median waiting times to points on the patient pathway. Introduction of the 2-week wait principle and the $\mathrm{DoH}$ guidelines for urgent referral has resulted in patients waiting longer for their first hospital visit (median of $7 v s 9$ days) and

Table I Number of referrals of suspected lung cancer

\begin{tabular}{|c|c|c|c|}
\hline & $\begin{array}{c}\text { Ist April } \\
\text { I999-3 Ist } \\
\text { March 2000, } \\
n(\%)\end{array}$ & $\begin{array}{c}\text { Ist April } \\
2000-3 \text { Ist } \\
\text { March 200 I, } \\
\text { n (\%) }\end{array}$ & $\begin{array}{c}\text { Ist April } \\
2001-3 \text { I st } \\
\text { March 2002, } \\
\text { n (\%) }\end{array}$ \\
\hline Total number of referrals & 286 & 352 & 404 \\
\hline 2-Week wait referral & $2(0.6)$ & $201(57)$ & $236(58)$ \\
\hline Urgent referral & $279(97.5)$ & $142(40)$ & $153(38)$ \\
\hline Other referral ${ }^{a}$ & $5(1.7)$ & $9(2.5)$ & $17(4.2)$ \\
\hline
\end{tabular}

longer for treatment of lung cancer. Time to diagnosis initially increased and then returned to preguideline values.

The proportion of patients who were seen within 2 weeks was $84 \%$ prior to the introduction of the ' 2 -week wait' scheme and $85 \%$ in the year after introduction of the 2 -week wait. The proportion fell to $71 \%(P<0.0001$; Mann-Whitney $U$-test $)$ in the year $2001-$ 2002. Analysis of the median waiting time by referral route demonstrated that all of those patients referred via the '2-week wait' scheme were seen within that time; however, as a result of the increase in absolute urgent referral numbers, the percentage of those referred by a conventional route who were seen within 2 weeks dropped significantly to $75.5 \%$ in $2000-2001$ and further to $60.9 \%(P<0.0001)$ in $2001-2002$.

\section{Reasons for referral}

The most common reasons for referral were cough, dyspnoea, weight loss, haemoptysis and chest pain. Table 3 shows the proportion of patients with each symptom by year, divided into patients with and without cancer. There was no difference in the pattern of symptoms after introduction of $\mathrm{DoH}$ guidelines. The frequency of these symptoms was found to be high in both patients with and without cancer. Chest pain, dyspnoea and weight loss were all more common in patients with lung cancer $(P<0.0001)$, but were still present in a large enough proportion of patients without cancer to mean they were not good discriminators. Haemoptysis was significantly more common in benign diagnoses $(P<0.0001)$.

An abnormal chest X-ray suggestive of lung cancer was the most common referral basis accounting for $87.2 \%$ over the 3 -year period. The finding of a mass was more common in cancer $(P<0.0001)$, but $30 \%$ of benign diagnoses also had a mass on chest radiograph. Consolidation and interstitial shadowing were more common in nonmalignant conditions $(P<0.0001)$.

Table 2 Effect of introduction of 2-week wait and DoH guidelines on waiting times

\begin{tabular}{|c|c|c|c|c|}
\hline & \multicolumn{3}{|c|}{ Waiting time in days: median (range) } & \multirow[b]{2}{*}{$P$-value } \\
\hline & $\begin{array}{c}\text { 1999-2000 (Department } \\
\text { of Health National } \\
\text { Standards, Local Action, } \\
\text { 2004) }\end{array}$ & $\begin{array}{c}\text { 2000-200I (Department } \\
\text { of Health NHS } \\
\text { Improvement Plan June, } \\
\text { 2004) }\end{array}$ & $\begin{array}{l}2001-2002 \\
\text { (National Institute } \\
\text { for Clinical } \\
\text { Excellence, 2005) }\end{array}$ & \\
\hline Referral to first hospital visit & $7(0-124)$ & $8(0-101)$ & $9(0-98)$ & 0.0009 for I vs 3 \\
\hline Referral to diagnosis & $26(0-228)$ & $33(2-307)$ & $27(0-300)$ & $<0.0000$ I for I vs $2 ; 0.0003$ for 2 vs 3 \\
\hline
\end{tabular}

$\mathrm{DoH}=$ Department of Health.

Table 3 Predominant symptoms of patients with and without cancer

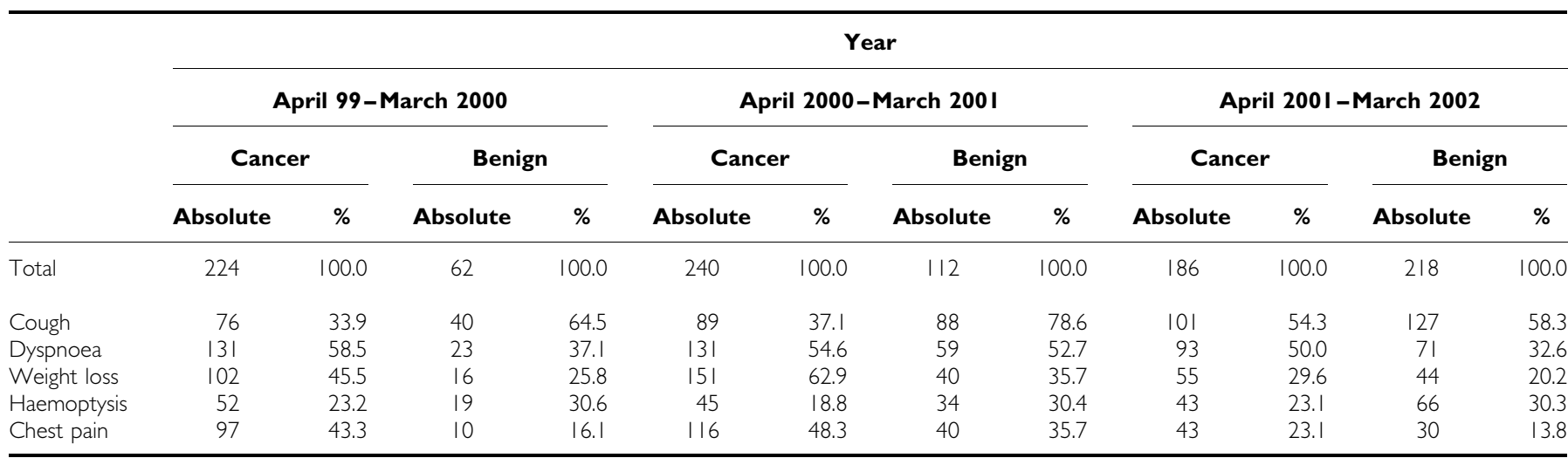


Table 4 Final diagnosis in all urgent referrals

\begin{tabular}{|c|c|c|c|c|c|c|}
\hline & \multicolumn{6}{|c|}{ Year } \\
\hline Total referrals & 286 & 100.0 & 352 & 100.0 & 404 & 100.0 \\
\hline All cancers & 224 & 78.3 & 240 & 68.2 & 186 & 46.0 \\
\hline SCLC & 37 & 12.9 & 30 & 8.5 & 19 & 4.7 \\
\hline Mesothelioma & 3 & 1.0 & 8 & 2.3 & 11 & 2.7 \\
\hline Carcinoid & I & 0.3 & I & 0.3 & 3 & 0.7 \\
\hline Lymphoma & 6 & 2.1 & 3 & 0.9 & 3 & 0.7 \\
\hline Metastatic & 16 & 5.6 & 25 & 7.1 & 18 & 4.5 \\
\hline No cancer & 62 & 21.7 & 112 & 31.8 & 218 & 54.0 \\
\hline
\end{tabular}

$\mathrm{NSCLC}=$ non-small-cell carcinoma; SCLC $=$ small-cell carcinoma.

Table 5 Stage of non-small-cell lung cancer

\begin{tabular}{llrll}
\hline & \multicolumn{4}{c}{ Stages } \\
\cline { 2 - 5 } Year (s) & $\mathbf{I}$ & $\mathbf{2}$ & $\mathbf{3}$ & $\mathbf{4}$ \\
\hline $1999-2000$ & 32 & 8 & 40 & 34 \\
$2000-2002$ & 48 & 23 & 79 & 54 \\
\hline
\end{tabular}

$\chi^{2}=2.51 ; P=0.47$

\section{Proportion of patients having cancer}

Table 4 shows the final diagnosis in all referrals. The proportion of urgent referrals subsequently found to have a malignancy decreased from $78 \%(224 / 286)$ prior to the introduction of the guidelines and the '2-week wait' scheme, to $68 \%(240 / 352)$ of patients in the first year $(P<0.0001)$ and $46 \%(186 / 404)$ of patients in the second year after their introduction $(P<0.0001)$.

\section{Stage of non-small-cell lung cancer}

Table 5 shows the number of patients in each surgical stage, where stage was recorded. In $1999-2000,75 \%$ of patients were staged, in $2000-2001,71 \%$ and in $2001-2002,77 \%$. There was no significant change in stage $\left(\chi^{2}, 2.52 ; P=0.47\right)$

\section{Benign diagnoses}

Table 6 shows the diagnostic categories for benign conditions referred as suspected lung cancer. Infections represented the largest increase in benign diagnoses. These cases were thought appropriate to refer to secondary care in 56,54 and $51 \%$ of patients in years 1999-2000, 2000-2001 and 2001-2002, respectively. In many other cases, however, it may have been appropriate on medical grounds alone to expedite a diagnosis by urgent referral, either because the condition required treatment or there was a high probability that there might be cancer.

\section{DISCUSSION}

Introduction of the target '2-week wait' scheme and DoH guidelines on urgent referral of suspected lung cancer has so far failed to achieve its primary objective of reducing waiting times. The data generated by this paper suggest two linked explanations for this unexpected finding: firstly, despite the publication of
Table 6 Benign diagnoses in patients referred urgently for suspected lung cancer

\begin{tabular}{lccc}
\hline & \multicolumn{3}{c}{ Frequency } \\
\cline { 2 - 4 } Condition & $\mathbf{1 9 9 9 - 2 0 0 0}$ & $\mathbf{2 0 0 0} \mathbf{- 2 0 0 1}$ & $\mathbf{2 0 0 1} \mathbf{- 2 0 0 2}$ \\
\hline Conditions that can usually be managed in primary care \\
Infection & 18 & 41 & \\
COPD & 3 & 3 & 83 \\
Goitre & 3 & 1 & 7 \\
Normal variant & 1 & 1 & 0 \\
ENT cause & 1 & 3 & 9 \\
CCF & 1 & 2 & 5 \\
& & & 2 \\
Conditions usually managed by respiratory physicians (or complex) & \\
Vascular markings & 3 & 4 & 3 \\
TB (old or active) & 3 & 7 & 4 \\
Benign lung tumour & 1 & 0 & 5 \\
Benign pleural shadows & 5 & 6 & 17 \\
Sarcoidosis & 1 & 3 & 2 \\
Benign lung shadow & 5 & 0 & 15 \\
Interstitial lung disease & 4 & 19 & 9 \\
Bronchiectasis & 3 & 9 & 13 \\
Other & 0 & 4 & 13 \\
DNA, died or refused tests & 4 & 6 & 10 \\
No diagnosis & 6 & 3 & 21 \\
Total & 62 & 112 & 218 \\
\hline COPD chron & & & \\
\hline
\end{tabular}

$\mathrm{COPD}=$ chronic obstructive pulmonary disease; $\mathrm{TB}=$ tuberculosis.

national guidelines recommending urgent referral through the '2week wait' scheme and local efforts to encourage this by simplifying referral procedures with proforma-based faxed referral letters/electronic referrals, a significant proportion of patients continued to be referred by standard means. Only $57.5 \%$ of patients were referred using the recommended '2-week wait' scheme. $37.7 \%$ were referred urgently via conventional means.

Secondly, there has been an increase of $42 \%$ in the number of urgent referrals for suspected lung cancer. This may reflect increased awareness of the problem in primary care, the perception of improved access to specialist teams or the inflexibility resulting from specific guidelines. The latter is suggested by the clear implication in some of the referral letters of doubt about whether the patient should be seen by a specialist. There may also have been a tendency to abuse the rapid access to specialist advice, although most patients still fitted DoH criteria. 
All of these factors may have resulted in the finding that the proportion of referrals culminating in malignant diagnoses has decreased from 78 to $46 \%$ in the 2 years since the guidelines were implemented.

These linked observations (more referrals with lower proportion of malignancy and a significant proportion of patients referred outside the 2-week wait system) have two important consequences: firstly that assessment of increasing numbers of patients with benign disease will place further strain on the lung cancer services, and secondly that the patients referred outside the 2 -week wait will be disadvantaged.

The first of these consequences is demonstrated in this paper by the increase in median time to first clinic visit from 7 to 9 days despite improvements in the efficiency of the referral process (including the appointment of an additional consultant). The second is illustrated by the finding that the waiting times were longer for non-2-week wait referrals. The increased number of referrals may have a beneficial influence on earlier diagnosis of lung cancer, but this was not supported by the findings of this study, as the stage did not change significantly. Moreover, making an earlier diagnosis may improve survival but not necessarily mortality - an effect of lead-time bias.

Thus, introduction of the guidelines and target wait scheme has had a beneficial effect for those patients referred by the recommended route, but a negative effect on those referred urgently outside this system. Furthermore, waiting times reported to the Government are based on patients referred through the 2 -week wait system and thus Government figures on waiting times for lung cancer do not reflect the true statistics. This may also apply to other cancers. Although out-with the scope of this paper, these data do prompt speculation that waiting times for urgent and

\section{REFERENCES}

Department of Health National Standards, Local Action (2004) Health and Social Care Standards and Planning Framework

Department of Health NHS Improvement Plan June (2004)

National Institute for Clinical Excellence (2005) The Diagnosis and Treatment of Lung Cancer, National Collarborating Centre for Acute Care Feb 2005. www.nice.org.uk routine noncancer referrals seen by the same consultants might also have increased as a consequence of the rapid increase in suspected cancer patients referred since the introduction of the guidelines.

The observed increase in early review of patients without cancer does have beneficial effects. The reassurance provided by rapid diagnosis and removal of a lengthy period of uncertainty is important for the individual. Furthermore, the study showed that over $50 \%$ of patients without cancer should probably have been in a respiratory medicine clinic in any case irrespective of cancer referral guidelines. Thus, the extra resources have had other ancillary benefits.

Despite the benefits of seeing more patients quickly, if the proposed Government targets of reducing the delay from referral to treatment to no more than 2 months by December 2005 (NHS Executive, 2000; National Institute for Clinical Excellence, 2005) are to be achieved, it is important that accurate baseline statistics are recorded. If the increase in referrals continues, considerable further investment in cancer services will be required and referral guidelines may need to be modified to reflect the management of minor haemoptysis. Currently, NICE (NICE, 2005) recommend offering the patient a chest radiograph prior to referral in patients presenting with haemoptysis. So far, it cannot be recommended that such patients are followed with a simple radiograph, but direct access to CT scanning and possibly bronchoscopy could reduce the burden on outpatient appointments while still identifying those patients with benign disease who may need a respiratory physician. Lastly, general practitioners should be aware that not using the 2-week wait system gives inaccurate national waiting time data and more importantly may disadvantage their patients.
NHS Executive (2000) Referral Guidelines for Suspected Cancer. London: Department of Health

NHS Executive (1997) The New NHS - Modern, Dependable. London: Department of Health (CM3807) 